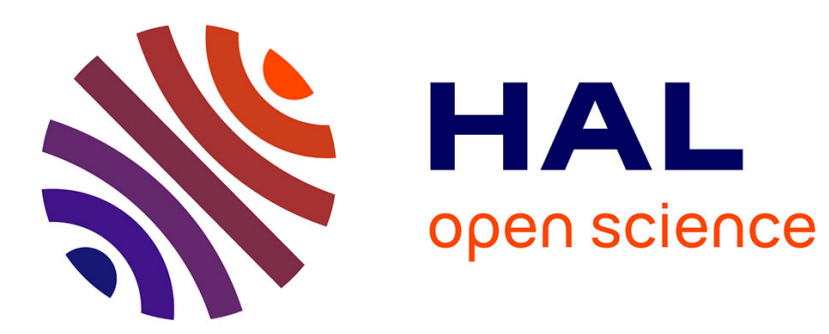

\title{
Field induced disruption of a planar water-oil interface influenced by a close metallic sphere
}

\author{
Pierre Atten, Jonathan Raisin, Jean-Luc Reboud
}

\section{To cite this version:}

Pierre Atten, Jonathan Raisin, Jean-Luc Reboud. Field induced disruption of a planar water-oil interface influenced by a close metallic sphere. International Conference on Dielectric Liquids (ICDL2008), Jun 2008, Poitiers, France. hal-00372099

\section{HAL Id: hal-00372099 \\ https://hal.science/hal-00372099}

Submitted on 31 Mar 2009

HAL is a multi-disciplinary open access archive for the deposit and dissemination of scientific research documents, whether they are published or not. The documents may come from teaching and research institutions in France or abroad, or from public or private research centers.
L'archive ouverte pluridisciplinaire HAL, est destinée au dépôt et à la diffusion de documents scientifiques de niveau recherche, publiés ou non, émanant des établissements d'enseignement et de recherche français ou étrangers, des laboratoires publics ou privés. 


\title{
Field Induced Disruption of a Planar Water-Oil Interface Influenced by a Close Metallic Sphere
}

\author{
P. Atten, J. Raisin, J.-L. Reboud \\ G2Elab, CNRS, Univ. Joseph Fourier and INP Grenoble, \\ BP 166, 38042 Grenoble Cedex 9, France
}

\begin{abstract}
The problem of instability of a horizontal interface between water and an insulating fluid, electrically influenced by a metallic sphere located just above it, is considered here with its relevance to the basic electrocoalescence phenomenon of close water droplets suspended in an insulating medium. Results are presented of preliminary experiments performed using visualisation and shadowscopy techniques. The evolution of the interface shape is characterised for various applied step voltages and for different values of the initial spacing $s_{0}$ between sphere and interface. An order of magnitude analysis leads to good estimates of the characteristic parameters and time scales.
\end{abstract}

\section{INTRODUCTION}

In the electrocoalescence phenomenon, the mutual attraction between two close drops first induces a quasi-static deformation of the facing interfaces [1]; in the critical conditions characterised by a finite spacing between interfaces, we guess that there is an instability leading to a fast deformation and eventually to the contact between the liquid of the two drops. Observations on droplets of medium size $(\sim 300 \mu \mathrm{m})$ using a fast camera suggest that this instability process occurs in a rather short time $<100 \mu \mathrm{s}$ [2]. The analogy with the exploding instability of a planar interface subjected to a uniform field [3] and with some observations in the electro wetting phenomenon [Mugele] suggests that there is a tendency to formation of a kind of spike.

In order to gain some insight on the fast deformation and disruption of the interfaces subjected to electric fields, we consider here a model experiment of a horizontal water layer influenced by a metallic sphere located at some distance above the interface. The high enough sphere-interface spacing $(\sim 1$ $\mathrm{mm}$ ) makes it possible to observe the interface shape. Preliminary results are presented.

\section{EXPERIMENTAL SETUP}

The test cell is a rectangular tank with four vertical Plexiglas walls to allow easy visualisation. Deionised water fills a cylindrical hole $(\phi=8 \mathrm{~cm})$ and we can add a synthetic polybutene oil as dielectric fluid. A grounded metallic sphere $(\phi=8 \mathrm{~mm})$ welded on a thin rod is hanging at a controllable level above the water surface [ICDL05]. High step voltage is applied to the water (a rectangular signal of duration $=40 \mathrm{~ms}$ delivered by a low frequency generator is amplified by a 20 $\mathrm{kV}$ high voltage amplifier - TREK Model 20/20C).

The cell is illuminated by a parallel light through a flash given by a LED and the magnified image is formed on photocathode of a CCD camera; the signal is digitized and recorded on a PC. The light flash is triggered by the sudden drop of the voltage applied to the water electrode either by the prescribed voltage pulse drop or by the water-sphere contact inducing a short-circuit.

A second optical arrangement was also used with a steady illumination; the magnified image is then formed on a plane with a narrow slit; the total light passing through the slit is measured by a photodiode. When the slit is located along the symmetry axis of the system, we recover information on the vertical rise of the water interface (the water absorbs light see Fig. 1).

\section{DEFORMATION OF WATER-AIR INTERFACE}

The problem which would correspond to the coalescence stage under the action of the field should start from a static deformation of the interface in conditions very close to the critical ones. This is not easy as a marked deformation is very sensitive to perturbations and its control is difficult. In this preliminary study, we worked in conditions where the voltage is suddenly applied and we examine how the interface evolves starting from the initial planar shape. We first considered the case of air-water interface.

Fig. 1, relative to the initial spacing $s_{0}=0.6 \mathrm{~mm}$, illustrates the fact that applying a voltage $V_{a p p l}$ during $40 \mathrm{~ms}$ induces a limited deformation for $V_{a p p l}<800 \mathrm{~V}$. For $V_{a p p l}>900 \mathrm{~V}$ the interface shape becomes very sensitive to the voltage value

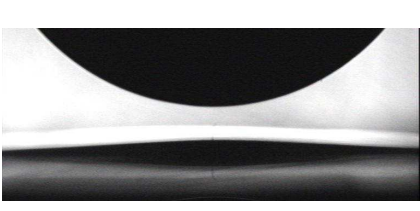

$800 \mathrm{~V}$

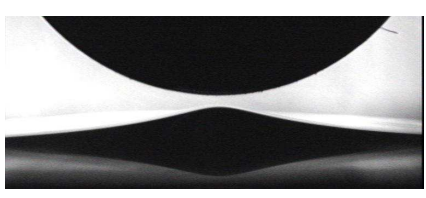

$940 \mathrm{~V}$

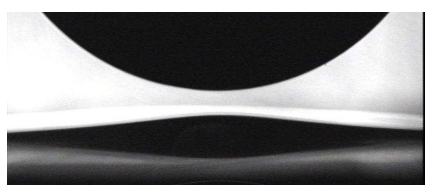

$900 \mathrm{~V}$

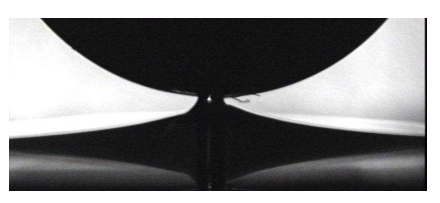

$950 \mathrm{~V}$
Fig. 1. Photographs (taken at $t=40 \mathrm{~ms}$ ) of the deformed water-air interface for various applied voltages $\left(s_{0}=0.6 \mathrm{~mm}\right)$. 
and its curvature at the axis is more and more important as $V_{\text {appl }}$ is raised. The marked change in shape for very small voltage increase is induced by the rather fast uprising of the interface when the instantaneous spacing $s$ between the water surface and the metallic sphere becomes small. This is due to the high field strength $\left(\sim V_{a p p l} / s\right)$ and the subsequent high electrostatic pressure at the apex of the deformed interface.

The fast final rise of the interface is clearly characterized by using the shadowscopy technique. In the oscillograms of Fig. 2 , the upper curves correspond to the applied voltage (the marked drop at $t<40 \mathrm{~ms}$ is due to the sphere-water contact) and the lower curves can be viewed as giving the time variation of the level of the interface on the system axis (the apparent stay of this level during the first $5 \mathrm{~ms}$ is due to imperfections in the optical arrangement). We see that, up to about $0.6 s_{0}$, the rising interface velocity is close to $1 \mathrm{~cm} / \mathrm{s}$; the final stage is much faster and the velocity is $\sim 20 \mathrm{~cm} / \mathrm{s}$.

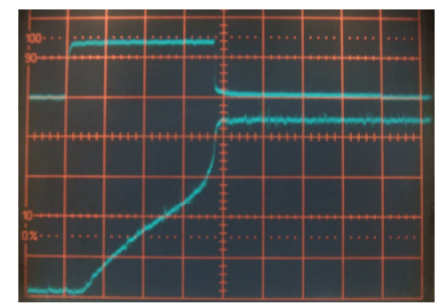

$960 \mathrm{~V}(10 \mathrm{~ms} / \mathrm{cm})$

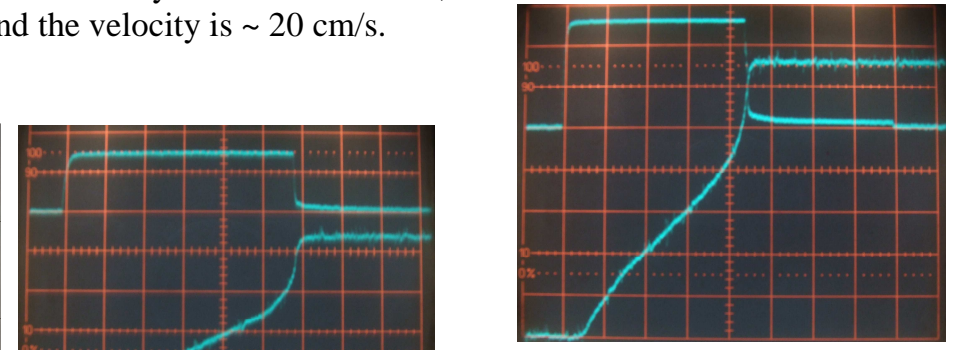

An extra phenomenon when working with air as insulating fluid is the occurrence of discharges in the gas in appropriate voltage and spacing conditions. Such discharges which play but a minor role for small initial spacing $s_{0}$ (for instance $s_{0}=$ $0.6 \mathrm{~mm}$ ) induce serious disturbances for larger $s_{0}$ values as can be seen on Fig. $3\left(s_{0}=1 \mathrm{~mm}\right)$. The discharge which occurs before the water reaches the sphere suppresses the electrostatic pressure and the subsequent evolution of the interface shape is mainly controlled by capillary waves (see the photographs for $V_{a p p l}=1560$ and $1600 \mathrm{~V}$ in Fig. 3). The picture taken at $t=25$ ms for $V_{a p p l}=1800 \mathrm{~V}$ (Fig. 3) does not exhibit such a clear disturbance because the instantaneous spacing is higher $\left(V_{a p p l}\right.$ is higher) and the interface deformation is at a earlier stage (note in Fig. 4 that the last stage of interface uprising for this voltage is slower than for $V_{a p p l}=1540 \mathrm{~V}$ ).
$1540 \mathrm{~V}(10 \mathrm{~ms} / \mathrm{cm})$

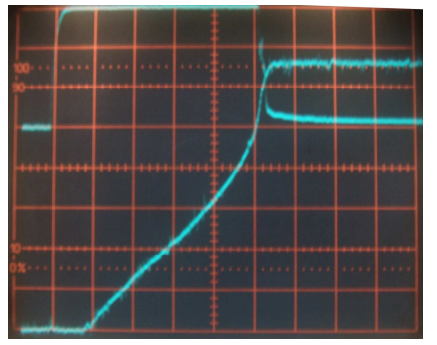

$1800 \mathrm{~V}(5 \mathrm{~ms} / \mathrm{cm})$
Fig. 4. Oscillograms given by the shadowscopy technique : applied voltage (upper curve) and approximate rise (lower curve) of the apex of the water-air interface $\left(s_{0}=1 \mathrm{~mm}\right)$.

\section{CASE OF WATER-OIL InTERFACE}

When working with oil as insulating medium, the electrical discharges are eliminated. For small gaps $s_{0}$, the deformation of the water-oil interface is qualitatively analogous to the one of water-air interface (see Figs. 5 and 1). Increasing the initial spacing $s_{0}$ results in a more and more marked bending of the interface (Figs. 6 and 7). We also observed that the deformation around the axis is sharper and sharper (see the example of Fig. 6 for $V_{a p p l}=722 \mathrm{~V}$ ).

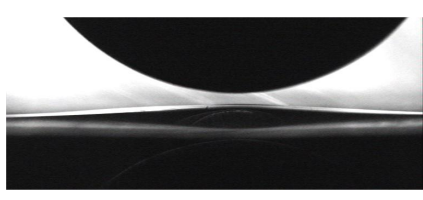

$240 \mathrm{~V}$

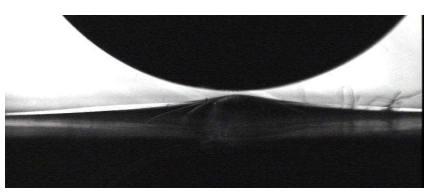

$274 \mathrm{~V}$

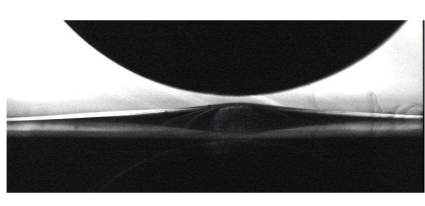

$268 \mathrm{~V}$

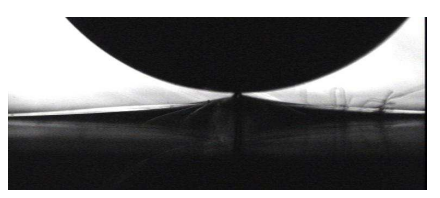

$276 \mathrm{~V}$
Fig. 5. Photographs $(t=40 \mathrm{~ms})$ of the deformed water-oil interface for various applied voltages $\left(s_{0}=0.4 \mathrm{~mm}\right)$.
Fig. 3. Photographs (generally taken at $t=40 \mathrm{~ms}$ ) of the deformed water-air interface for various applied voltages $\left(s_{0}=1 \mathrm{~mm}\right)$. 

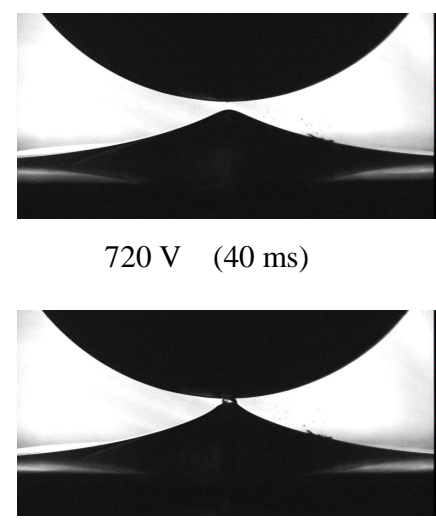

$750 \mathrm{~V}(38 \mathrm{~ms})$
$720 \mathrm{~V} \quad(40 \mathrm{~ms})$

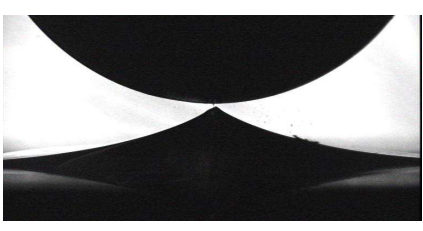

$722 \mathrm{~V}(40 \mathrm{~ms})$

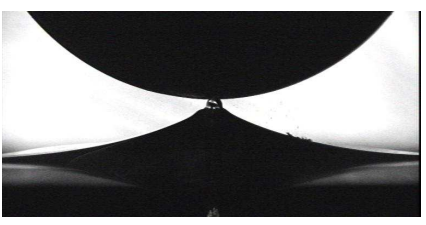

$800 \mathrm{~V} \quad(29 \mathrm{~ms})$
Fig. 6. Photographs of the deformed water-oil interface for various applied voltages in the case $s_{0}=1 \mathrm{~mm}$.

For an applied voltage high enough, the interface impacts the metallic sphere at a time lower than the predetermined duration $(40 \mathrm{~ms})$ of the voltage pulse. Photographs taken shortly after the water-sphere contact reveal an interface shape to a first approximation independent of the applied voltage (Figs. 6 and 7). This suggests that the voltage amplitude affects the dynamics of the interface deformation and of the associated motions of water and oil but not the shape at the successive stages of interface uprising (at least to a first approximation). By closely examining the zones near the water-sphere "contact", it appeared that the short ligament has a diameter increasing rapidly with time because of the flux of
$1600 \mathrm{~V} \quad(40 \mathrm{~ms})$

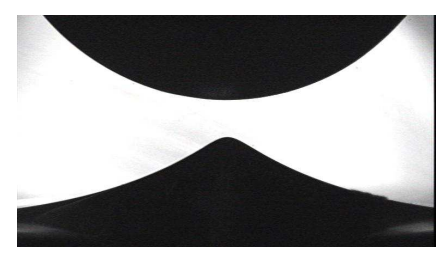

$1720 \mathrm{~V} \quad(40 \mathrm{~ms})$

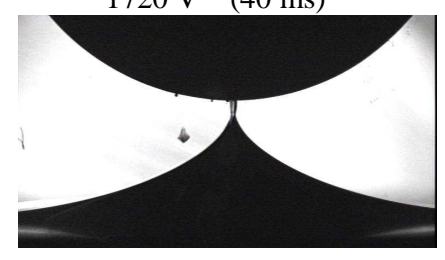

$1900 \mathrm{~V} \quad(34 \mathrm{~ms})$

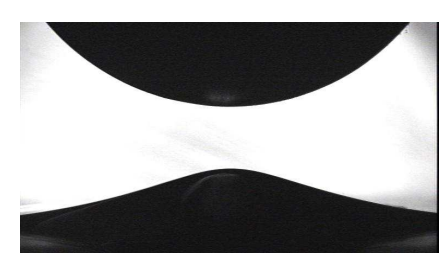

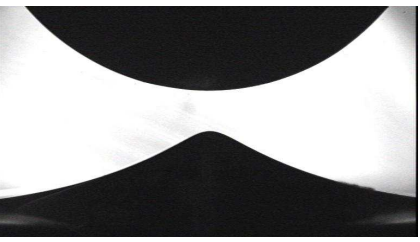

$1700 \mathrm{~V} \quad(40 \mathrm{~ms})$

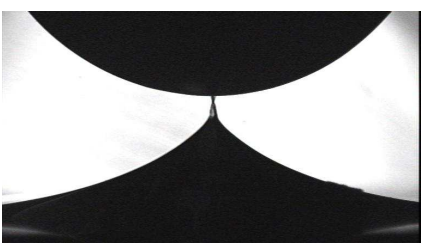

$1730 \mathrm{~V} \quad(40 \mathrm{~ms})$

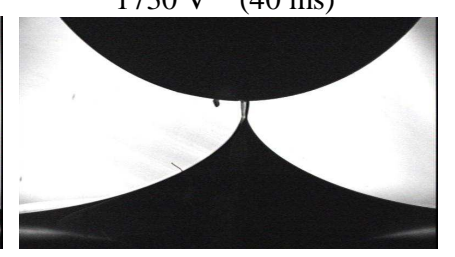

$2000 \mathrm{~V} \quad(32 \mathrm{~ms})$
Fig. 7. Photographs of the deformed water-oil interface for various applied voltages in the case of a rather large gap $s_{0}=2 \mathrm{~mm}$.

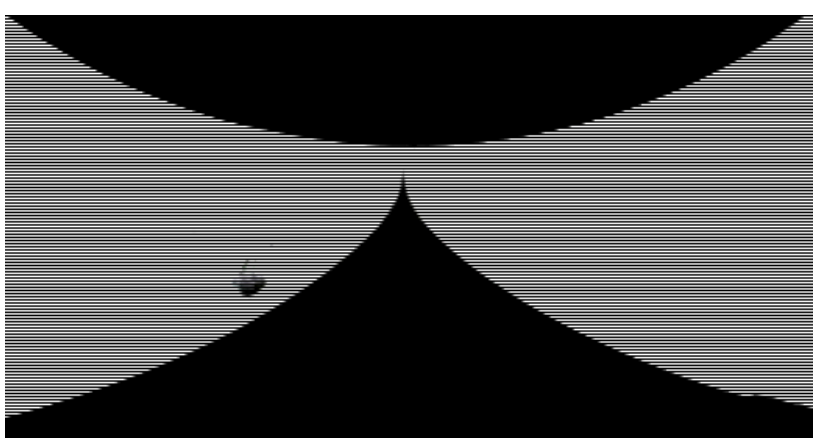

$1750 \mathrm{~V}$

(40 ms)

Fig. 8. Photograph of the disrupting water-oil interface just before the contact between water and metallic sphere $\left(s_{0}=2 \mathrm{~mm}\right)$.

uprising water. it can then be inferred that the last stage of interface deformation is a transition toward a jet presumably having a high velocity. After numerous trials we obtained the - magnified - picture of Fig. 8 unambiguously showing a very thin jet emitted by an elongating cone-like zone. Attempts to visualise such a jet in the case $s_{0}=1 \mathrm{~mm}$ were unsuccessful, very likely because the reproducibility is not very good (after each voltage pulse the sphere is removed, cleaned and repositioned). In any case the question arises concerning the occurrence of such a jet-like disruption of the interface for small values of the initial spacing $s_{0}$.

\section{ORdER OF MAGNITUdE ANALYSIS}

A very rough model consists in following the rise of a certain amount of water (and oil) under the action of the electric forces. The time evolution is governed by the basic equation :

$$
M \frac{d w}{d t}=F_{e s}-F_{g}-F_{c a p}
$$

where $M$ stands for the mass of liquid, $w$ for the interface velocity on the axis and $F_{e s}, F_{g}$ and $F_{c a p}$ are the magnitudes of electrostatic, gravitational and capillary forces.

The electrostatic pressure applies on an effective area decreasing as the water level rises; it has been shown that the pertinent scale then is $R_{\text {eff }}=\left(R_{0} s\right)^{1 / 2}$ [6] where $R_{0}$ is the sphere radius and $s=s_{0}-z_{\text {int }}$ the minimum distance between water and sphere $\left(z_{\text {int }}\right.$ denotes the height of the interface apex and $w$ $\left.=d z_{\text {inl }} / d t\right)$. With this scale we deduce $F_{\text {es }} \sim(\pi / 2) R_{\text {eff }}^{2} \varepsilon V_{\text {appl }}{ }^{2} / s^{2}$ ( $\varepsilon$ : fluid permittivity). The extra pressure due to the interface rise is $\Delta \rho g z_{\text {int }}$ where $\Delta \rho$ is the density difference between the two fluids; the area concerned by the interface rise does not significantly change during the interface rising and we take $F_{g} \sim \pi R_{0} s_{0} \Delta \rho g z_{\text {int }}$. At the axis the capillary pressure due to the interface curvature is $2 T / R$ where $T$ is the surface tension and $R$ the radius of curvature; as the zone of strong curvature is closely related to the zone of high electrostatic pressure, we have $F_{c a p} \sim 2 \pi R_{\text {eff }}{ }^{2} T / R$. We can link $R$ with $z_{\text {int }}$ by stating that, in the vicinity of the axis, the interface has a parabolic shape with the parabola intersecting the plane $z=0$ 
at a distance $x_{0}$ from the axis ; this leads to $1 / R=z_{\text {int }} / x_{0}^{2}$. Taking $x_{0}=\alpha^{1 / 2} R_{\text {eff }}$ ( $\alpha$ : numerical constant) finally gives $F_{c a p}$ $\sim(2 \pi / \alpha) T z_{\text {int }}$.

The deformation and rise of the interface implies the motion of a volume of liquid that we assume to be again characterised by the length scale $R_{\text {eff }}$ so that $M=\beta \rho_{\text {water }} R_{\text {eff }}^{3}$, $\beta$ being a constant. By introducing the relative height $f_{0}$ of the interface $\left(f_{0}=z_{\text {int }} / s_{0}\right)$, equation (1) can be rewritten as follows:

$$
\frac{d^{2} f_{0}}{d t^{2}}=\frac{1}{\tau^{2}}\left[B e \frac{1}{\left(1-f_{0}\right)^{5 / 2}}-\left(\frac{2}{\alpha}+B o\right) \frac{f_{0}}{\left(1-f_{0}\right)^{3 / 2}}\right]
$$

The non dimensional Bond number $B o$ and electrical Bond number $B e$ have the expressions [5] :

$$
B o=\frac{\Delta \rho g R_{0} s_{0}}{T} \quad B e=\frac{1}{2} \frac{\varepsilon V_{a p p l}{ }^{2} R_{0}}{T s_{0}{ }^{2}}
$$

and the time constant $\tau$ defined by :

$$
\tau=\left[\frac{\beta}{\pi} \frac{\rho_{\text {water }} R_{0}^{3 / 2} s_{0}^{3 / 2}}{T}\right]^{1 / 2}
$$

is the capillary time built with the length scale $R_{\text {eff. }}$ Its typical value in the case $R_{0}=4 \mathrm{~mm}$ and $s_{0}=1 \mathrm{~mm}$ is on the order of $10^{-2} \mathrm{~s}$. Of course the time scale of the problem also depends on the applied voltage through the parameter $B e$ but, as the $B e$ values typically range from 0.2 to 0.6 in the conditions of interest here, this does not change the order of magnitude of the effective time scale.

Static solutions for the interface deformation correspond to the right hand side of (2) being equal to zero and can be obtained only for small enough $B e$ values. The maximum of $B e$ corresponds to $\left(f_{0}\right)_{\text {crit }}=0.5$, which leads to $B e_{\text {crit }} \cong 1 /(2 \alpha)+$ $B o / 4$, an estimate in fair agreement with theoretical and experimental results [5] when taking $\alpha \cong 2$. Considering the

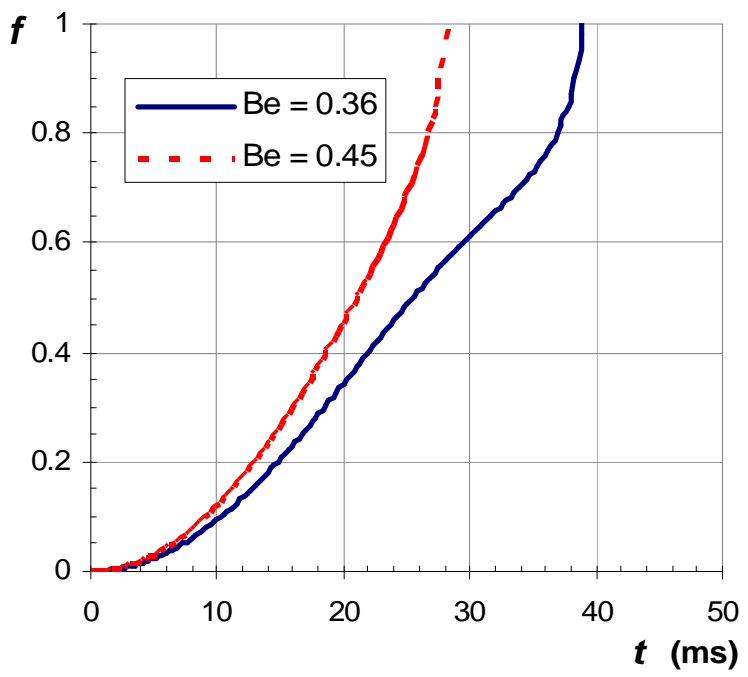

Fig. 9. Variation with time of the normalized level of the apex of water-air interface (solution of (2) in the case $\alpha=2, B o=0.56$ and $\tau \cong 13 \mathrm{~ms}$ ). case of water-air interface for an initial spacing $s_{0}=1 \mathrm{~mm}$, integration of (2) with the constants $\alpha=2$ and $\beta=5$ gives results as shown in Fig. 9 (in this model the critical voltage is defined by $B e_{\text {crit }}=0.39$, a value close to the measured ones [5]). The two curves shown in Fig. 9 are qualitatively similar to the oscillograms of Figs. 2 and 4. The mean rising velocity $(\sim 2 \mathrm{~cm} / \mathrm{s})$ for the two curves of Fig. 9 is a fairly good estimate for the first stage ( $f_{0}$ up to about 0.6 ) of interface deformation as shown by Fig. 4. However, this model does not predict the fast final uprising. This is not surprising because for an interface which sharpens and tends to emit a jet, the very rough estimations of the various forces and of $M$ are not valid.

\section{Discussion AND CONCLUSIONS}

These preliminary experiments have shown that for a large enough spacing $s_{0}$ the water-oil interface raises and deforms tending to a more or less conical shape; in the last stage of the apex rising, due to the very strong electrostatic pressure there is a strong acceleration of the motion associated with the transition towards a jet. Although this has not been visualised unambiguously, we guess that a similar phenomenon of interface disruption occurs for small gaps. A refined technique of visualisation is under development to investigate the last stage of interface disruption and to characterise the length scale of jet-like formation.

\section{ACKNOWLEDGMENT}

This work is developed in the framework of a collaborative research between the French CNRS and the Consortium working on the project "Electrocoalescence - Criteria for an efficient process in real crude oil systems"; co-ordinated by SINTEF Energy Research. Contact person is L.E. Lundgaard. The project is supported by The Research Council of Norway, under the contract no: 169466/S30, and by the following industrial partners: Vetco Aibel AS, Aker Kvaerner Process Systems AS, StatoilHydro ASA, BP Exploration Operating Company Ltd, Shell Technology Norway AS, Petrobras.

\section{REFERENCES}

[1] P. Atten, "Critical conditions for electrically induced coalescence of two very close water droplets in oil", Proceed. ICDL 2005, IEEE-Cat.-No. 05CH37643, pp. 177-180, 2005.

[2] L.E. Lundgaard and G. Berg, private communication.

[3] G. Neron de Surgy, J.-P. Chabrerie, "Emission of liquid metal in vacuum”, IEEE Trans. DEI, vol. 2 (2), pp. 184-189, 1995.

[4] F. Mugele, J.-C. Baret, "Electrowetting: from basics to applications", J. Phys.: Condens. Matter, 17, pp. R705-R774, 2005.

[5] P. Atten, F. Aitken, D. Koulova-Nenova, "Field-induced deformation and disruption of a planar water-oil interface under the influence of a conducting sphere", Proceed. ICDL 2005, IEEE-Cat.-No. 05CH37643, pp. 165-168, 2005.

[6] P. Atten, L.E. Lundgaard and G. Berg, "A simplified model of electrocoalescence of two close water droplets in oil", J. of Electrostatics, vol. 64, pp. 550-554, 2006. 